\title{
ON ALGEBRAIC SURFACES WHICH ARE INVARIANT IN A CERTAIN CLASS OF FINITE COLLINEATION GROUPS*
}

\section{BY ARNOLD EMCH}

1. Introduction. Among the algebraic varieties in projective space of 5 dimensions, $S_{5}$, and its sub-spaces and projections, there exist examples with notable properties and historic interest.

There is the Veronese surface of order 4 in $S_{5}$ obtained by a mapping process based on the $\infty^{5}$ conics of a plane. The projection of this surface from an $S_{1}$ which has no point in common with the surface upon an $S_{3}$ is a Steiner surface, as is well known. Again we have Segre's cubic variety cut out from the hypersurface

$$
x_{1}^{3}+x_{2}^{3}+\cdots+x_{6}{ }^{3}=0
$$

by the hyperplane

$$
x_{1}+x_{2}+\cdots+x_{6}=0
$$

in $S_{5}$. The tangent hypercone from a generic point of this variety to the variety itself cuts a generic $S_{3}$ in a Kummer surface.

In what follows I shall consider a $V_{3}$ in $S_{5}$ defined as the intersection of two hyperquadrics in $S_{5}$. In coordinate representation this space shall be given by $S_{5} \equiv\left(y_{1}, y_{2}, \cdots, y_{6}\right)$ and the two hyperquadrics by

$$
\begin{aligned}
& y_{1} y_{4}-y_{2} y_{5}=0, \\
& y_{2} y_{5}-y_{3} y_{6}=0 .
\end{aligned}
$$

Obviously the $V_{3}$ determined by (1) and (2) is also contained in the hyperquadric $y_{1} y_{4}-y_{3} y_{6}=0$.

If to (1) and (2) the hyper $n$-ic

$$
y_{1}^{n}+y_{2}^{n}+\cdots+y_{6}^{n}=0
$$

* Presented to the Society, April 18, 1930. 
is adjoined, then the three intersect in a surface which is of order $3 n$ and may be represented by

(4) $y_{2} y_{3}^{n}\left(y_{1}^{n}+y_{2}^{n}+y_{3}^{n}+y_{4}^{n}\right)+y_{1}^{n} y_{4}^{n}\left(y_{2}^{n}+y_{3}^{n}\right)=0$.

The problem arises whether it is possible to reduce this surface birationally to a simpler form. This may indeed be accomplished by the following transformation of the space $S_{5}(y)$ into an ordinary space $S_{3}(x)$ :

$$
\begin{aligned}
& \rho y_{1}=x_{1} x_{4}, \quad \rho y_{2}=x_{2} x_{4}, \quad \rho y_{3}=x_{3} x_{4}, \\
& \rho y_{4}=x_{2} x_{3}, \quad \rho y_{5}=x_{3} x_{1}, \quad \rho y_{6}=x_{1} x_{2} \text {. }
\end{aligned}
$$

Clearly (1) and (2) are satisfied by (5). From this we find inversely

$$
\begin{aligned}
& \sigma x_{1}=1 / y_{4}, \quad \sigma x_{2}=1 / y_{5}, \quad \sigma x_{3}=1 / y_{6}, \\
& \sigma x_{4}=\left(y_{1}+y_{2}+y_{3}\right) /\left(y_{4} y_{5}+y_{5} y_{6}+y_{6} y_{4}\right),
\end{aligned}
$$

with the adjunction of (1) and (2), so that the correspondence is of the Cremona type.

Since (5) is a Cremona transformation satisfying (1) and (2), and since the hyper $n$-ic (3) is transformed by (5) into the surface

$$
x_{1}^{n} x_{2}^{n}+x_{2}^{n} x_{3}^{n}+x_{3}^{n} x_{1}^{n}+x_{1}^{n} x_{4}^{n}+x_{2}^{n} x_{4}^{n}+x_{3}^{n} x_{4}^{n}=0,
$$

an $F_{2 n}$ of $S_{3}$, the surface (4) defined by (1), (2) and (3) in $S_{5}$ is transformed birationally by (5) into the surface (7).

The problem is now resolved into an investigation of these surfaces $F_{2 n}$ of order $2 n$ in three dimensions.

2. Group Properties of Surface $F_{2 n}$. As (7) is symmetric in the $x$ 's, the surface is obviously invariant under the symmetric $G_{24}$ on four letters. But there exists a larger group of collineations containing the symmetric group as a subgroup. Denote the $n$ roots of unity by $1, r_{2}, r_{3}, \cdots, r_{n}$. Then as $\left(r_{k} x_{i}\right)^{n}=x_{i}{ }^{n}$, it is clear that

$$
\left(\begin{array}{cccc}
x_{1} & x_{2} & x_{3} & x_{4} \\
x_{1 r_{\alpha}} & x_{2 r_{\beta}} & x_{3 r_{\gamma}} & x_{4 r_{\delta}}
\end{array}\right)=\left(\begin{array}{cccc}
x_{1} & x_{2} & x_{3} & x_{4} \\
x_{1} & x_{2 r_{i}} & x_{3 r_{k}} & x_{4 r_{l}}
\end{array}\right)
$$

a substitution which leaves $F_{2 n}$ invariant. There are as many such substitutions as there are dispositions of $n$ elements with 
repetitions in 3 places, that is, $n^{3}$. Each of these can be permuted in 24 different ways, so that there exists a collineation group of order $24 \cdot n^{3}$ under which the surface is invariant. When $n$ is even, the octavic group

$$
\left(\begin{array}{cccc}
x_{1} & x_{2} & x_{3} & x_{4} \\
\pm x_{1} & \pm x_{2} & \pm x_{3} & \pm x_{4}
\end{array}\right)
$$

may be adjoined, so that in this case the surface is invariant under a group of order $8 \cdot 24 \cdot n^{3}$. The group of $n^{3}$ collineations is Abelian and is invariant in the entire group, since the transform of any of its substitutions is

$$
\left(\begin{array}{llll}
x_{1} & x_{2} & x_{3} & x_{4} \\
x_{1} & x_{2 r_{i}} & x_{3 r_{k}} & x_{4 r_{l}}
\end{array}\right) \text { by }\left(\begin{array}{llll}
x_{1} & x_{2} & x_{3} & x_{4} \\
x_{a} & x_{b} & x_{c} & x_{d}
\end{array}\right)
$$

$$
\left(\begin{array}{llll}
x_{a} & x_{b} & x_{c} & x_{d} \\
x_{a} & x_{b r_{i}} & x_{c r_{k}} & x_{d r_{l}}
\end{array}\right)
$$

The entire group is the direct product of the symmetric group on four elements and this Abelian invariant subgroup. The result may be stated as follows:

THEOREM 1. The symmetric surface $\sum x_{\imath}^{n} x_{k}=0, i \neq k$, is invariant under a collineation group of order $24 \cdot n^{3}$ or $8 \cdot 24 \cdot n^{3}$, according as $n$ is odd or even. The group is the direct product of the symmetric group $G_{24}$, or a group $G_{192}$ in case of an even $n$, with an invariant Abelian subgroup of order $n^{3}$.

3. Conics on the Surface. The surface may be written in the form

or

$$
\left(x_{2}^{n}+x_{3}^{n}\right)\left(x_{1}^{n}+x_{4}^{n}\right)+x_{1}^{n} x_{4}^{n}+x_{2}^{n} x_{3}^{n}=0
$$

$$
\begin{aligned}
& \Pi\left(x_{2}-e^{(1+2 k) i \pi / n} \cdot x_{3}\right) \cdot \Pi\left(x_{1}-e^{(1+2 k) i \pi / n} \cdot x_{4}\right) \\
& \quad+\Pi\left(x_{1} x_{4}-e^{(1+2 k) i \pi / n} \cdot x_{2} x_{3}\right)=0 ; k=0,1, \cdots, n-1 .
\end{aligned}
$$

The first product, set equal to zero, represents $n$ planes through the side $A_{1} A_{4}$ of the coordinate tetrahedron. The third product represents $n$ quadrics on the skew quadrilateral $A_{1} A_{2}, A_{2} A_{4}$, $A_{4} A_{3}, A_{3} A_{1}$. Hence there are $n$ planes through the side $A_{1} A_{4}$ which cut these quadrics in $n^{2}$ conics which lie on the surface $F_{2 n}$. The same is, of course, true for every other side of the coordinate tetrahedron. In each of these planes there are $n$ 
of these conics. When $n$ is odd, $n=2 m+1$, then for $k=m$ the corresponding plane through $A_{1} A_{4}$ becomes $x_{2}+x_{3}=0$, which cuts the quadric $x_{1} x_{4}+x_{2} x_{3}=0$ in a real conic. Hence, when $n$ is odd, the surface contains 6 real conics which lie on the quadric $\sum x_{i} x_{k}=0, i \neq k$. In this case the surface also passes through the points $(-1,1,1,1) ;(1,-1,1,1) ;(1,1,-1,1) ;(1,1,1,-1)$ which form a desmic tetratredron with respect to the coordinate tetrahedron. The four centers of perspective of these form the third desmic tetrahedron $(1,1,1,1) ;(-1,-1,1,1)$; $(-1,1,-1,1) ;(1,-1,-1,1)$, which is not on the surface. The points $A_{1}, A_{2}, A_{3}, A_{4}$ are obviously $n$-fold points of the surface. Hence we have the following theorem.

Theorem 2. The surface $F_{2 n}$ has $A_{1}, A_{2}, A_{3}, A_{4}$ as $n$-fold points and contains $6 n^{2}$ conics which lie 6 times on $n^{2}$ planes through the joins $A_{i} A_{k}$. On each of these planes lie $n$ of the conics. When $n$ is odd, then 6 of these conics are real and lie on the symmetric quadric through the four multiple points.

4. Lines on the Surface. If the equation of $F_{2 n}$ is written in the form

$$
x_{1}^{n} x_{2}^{n}+x_{2}^{n} x_{3}^{n}+x_{3}^{n} x_{1}^{n}+x_{4}^{n}\left(x_{1}^{n}+x_{2}^{n}+x_{3}{ }^{n}\right)=0
$$

it is clear that the $2 n^{2}$ lines of intersection of the two cones $x_{1}{ }^{n} x_{2}{ }^{n}$ $+x_{2}{ }^{n} x_{3}{ }^{n}+x_{3}{ }^{n} x_{1}{ }^{n}=0, x_{1}{ }^{n}+x_{2}{ }^{n}+x_{3}{ }^{n}=0$ lie on the surface. Thus is established the existence of $8 n^{2}$ lines, $2 n^{2}$ through each multiple point, on the surface. The equations of these lines may easily be established. Setting $x_{1}{ }^{n}=z_{1}, x_{2}{ }^{n}=z_{2}, x_{3}{ }^{n}=z_{3}$, the traces of the lines through $A_{4}$ for example are obtained as the solutions of $z_{1} z_{2}+z_{2} z_{3}+z_{3} z_{1}=0$ and $z_{1}+z_{2}+z_{3}=0$ or of $x_{1}{ }^{n}=1, x_{2}{ }^{n}=\omega$, $x_{3}{ }^{n}=\omega^{2}$, where $1, \omega, \omega^{2}$ are the cube roots of unity. These solutions may be written in the form

$$
\begin{aligned}
& x_{1}=e^{2 k i \pi / n}, x_{2}=e^{2 i \pi /(3 n)+2 k i \pi / n}, x_{3}=e^{4 i \pi /(3 n)+2 k i \pi / n}, \\
& x_{1}=1, x_{2}=e^{2 i \pi\left[3 n+3\left(k-k_{1}\right)+1\right] /(3 n)}, x_{2}=e^{2 i \pi\left[3 n+3\left(k-k_{1}\right)+2 k\right] /(3 n)}, \\
& k, k_{1}=0,1, \cdots, n-1, \text { hence also } k-k_{1}=0, \pm 1, \cdots, \pm(n-1) .
\end{aligned}
$$
Now we may distinguish three cases, according as $n \equiv 0,1,2$ $(\bmod 3)$, or $n=3 m, n=3 m+1, n=3 m+2$. When $n=3 m$, then it is impossible for $x_{2}$ or $x_{3}$ to become either $\omega$, or $\omega^{2}$, since $6 m+3\left(k-k_{1}\right)+1 \neq 0$ and also $6 m+3\left(k-k_{1}\right)+2 \neq 0$. This however is possible when $n=3 m+1$, by choosing $k_{1}=k=2 m+1$, to 
get $x_{2}=\omega$, and $k_{1}-k=m+1$ to get $x_{3}=\omega^{2}$. Likewise when $n=3 m+2$, for $k_{1}-k=m+1$, we have $x_{2}=\omega^{2}$, and for $k_{1}-k$ $=2(m+1), x_{3}=\omega$. Now $\left(1, \omega, \omega^{2}\right)$ and $\left(1, \omega^{2}, \omega\right)$ are the intersections of the unit line $x_{1}+x_{2}+x_{3}=0$ with the conic $x_{1} x_{2}+x_{2} x_{3}$ $+x_{3} x_{1}=0$, so that when $n=3 m+1$, or $3 m+2$, two of the $2 n^{2}$ lines through $A_{4}$ pass through these points in $x_{4}=0$. Two such lines pass through each of the remaining three vertices. The six traces of these lines lie on the unit-plane $x_{1}+x_{2}+x_{3}+x_{4}=0$ and are the intersections of the quadric $\sum x_{i} x_{k}=0, i \neq k$, with the quadrilateral cut out on the unit-plane by the faces of the coordinate tetrahedron. But when $n=3 m$, there are $18 \mathrm{~m}^{2}$ lines through each vertex whose traces on the opposite face lie by sixes on $3 m^{2}$ conics; i.e., the lines lie by sextuples on $3 m^{2}$ quadric cones with the same vertex.

These lines intersect by pairs in the following manner: Let $S=\left(1, s_{1}, s_{2}\right)$ be one of the $2 n^{2}$ solutions as represented above by $\left(x_{1}, x_{2}, x_{3}\right)$ in terms of exponentials. Then $\left(1, s_{1}, s_{2}, s_{3}\right)$ is a point on the line $A_{4} S$ which lies on the surface. If we now let $s_{3}$ take on the $3 n$ values $1, r_{2}, \cdots, r_{n} ; s_{1}{ }^{1}, s_{1}{ }^{2}, \cdots, s_{1}{ }^{n} ; s_{2}{ }^{1}$, $s_{2}{ }^{2}, \cdots, s_{2}{ }^{n}$ corresponding to the $3 n$ solutions for $x_{1}{ }^{n}=1$, $x_{2}{ }^{n}=\omega, x_{3}{ }^{n}=\omega^{2}$ separately, we obtain $3 n$ points on $A_{4} S$ through each of which pass two lines of the surface. For example through $\left(1, s_{1}, s_{2}, s_{1}{ }^{i}\right)$ pass the lines joining $A_{4}$ to $\left(1, s_{1}, s_{2}, 0\right)$ and $A_{2}$ to $\left(1,0, s, s_{1} i\right)$. Thus there exist $3 n$ lines of the surface which intersect a line $A_{4} S$ of the surface, such that through each vertex $A_{1}, A_{2}, A_{3}$ pass $n$ of the 3 lines. Hence on account of the symmetry there exist $\frac{1}{2} \cdot 3 n \cdot 2 n^{2} \cdot 4=12 n^{3}$ points on the surface through each of which pass two lines of the surface. This may be summed up as follows.

THEOREM 3. There exist $8 n^{2}$ lines on the surface, $2 n^{2}$ through each multiple point. These lines intersect by pairs in $12 n^{3}$ points of which $3 n$ lie on each line. In case $n=3 m$, the lines through each point $A_{i}$ lie by sixes on $3 \mathrm{~m}^{2}$ cones with the common vertex $A_{i}$. In case $n=3 m+1$ there are $3 m^{2}-2 m$, in case $n=3 m+2$, there are $3 m^{2}+4 m+2$ such cones. The configuration of $12 n^{3}$ points is, of course, invariant under the finite collineation group of the surface.

The surface $F_{2 n}$ is also invariant under the involutorial cubic transformation 
$\rho x_{1}^{\prime}=x_{2} x_{3} x_{4}, \quad \rho x_{2}^{\prime}=x_{1} x_{3} x_{4}, \quad \rho x_{3}^{\prime}=x_{1} x_{2} x_{4}, \quad \rho x_{4}^{\prime}=x_{1} x_{2} x_{3}$.

From this it follows that $F_{2 n}$ is invariant in a group of Cremona transformations whose order is twice the order of the corresponding collineation group.

5. Example. The Sextic Surface $\sum x_{i}{ }^{2} x_{k}{ }^{2}=0, i \neq k$. This surface assumes a particularly attractive form by the following transformation from projective into Cartesian coordinates. Choose a cube $A_{1}(-1,1,1) ; A_{2}(1,-1,1)$; $A_{3}(1,1,-1) ; A_{4}(-1,-1,-1) ; A_{1}^{\prime}(1,-1,-1) ; A_{2}{ }^{\prime}(-1,1,-1)$; $A_{3}{ }^{\prime}(-1,-1,1) ; A_{4}{ }^{\prime}(1,1,1)$, so that the Cartesian axes $O X$, $O Y, O Z$ are the lines joining the centers of opposite faces of the cube. Now let the coordinate tetrahedron of the projective space $\left(x_{1}, x_{2}, x_{3}, x_{4}\right)$ coincide with $A_{1} A_{2} A_{3} A_{4}$; then

$$
\begin{array}{ll}
\rho x_{1}=-x+y+z+1, & \rho x_{2}=x-y+z+1, \\
\rho x_{3}=x+y-z+1, & \rho x_{4}=-x-y-z+1 .
\end{array}
$$

The surface $F_{6}$ reduces to

$$
\begin{gathered}
(-x+y+z+1)^{3}(x-y+z+1)^{3}+(x-y+z+1)^{3}(x+y-z+1)^{3} \\
+(x+y-z+1)^{3}(-x+y+z+1)^{3} \\
+(-x-y-z+1)^{3}\left\{(-x+y+z+1)^{3}\right. \\
\left.+(x-y+z+1)^{3}+(x+y-z+1)^{3}\right\}=0 .
\end{gathered}
$$

The quadric $\sum x_{i} x_{k}=0, i \neq k$, reduces to the sphere $x^{2}+y^{2}+z^{2}-3$ $=0$, so that the six real conics on the sextic surface become circles which lie in the faces of the cube and which in each case pass through the four vertices of the cube in this face. The unit plane is transformed into the plane at infinity and the three desmic tetrahedra become the two regular tetrahedra inscribed in the cube and the tetrahedron formed by the origin and the infinite points of the $x$-, $y_{-}$, and $z$-axis. There are 72 lines on the sextic. The 18 lines through each vertex lie by sixes on 3 coaxial right circular cones with the principal diagonals of the cube as axes. The collineation group under which the surface is invariant is of order $24 \cdot 27=648$. It also permutes the points of the configuration of 324 points through each of which pass 2 of the 72 lines. On each line there are 9 of these points.

The UnIVERsity of ILLiNoIS 